Canadian Journal of Civil Engineering

Canadian

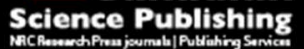
Revue canadienne de génie civil

\title{
An Alternative Roughness Index to IRI for Flexible Pavements
}

\begin{tabular}{|r|l|}
\hline Journal: & Canadian Journal of Civil Engineering \\
\hline Manuscript ID & cjce-2017-0443.R1 \\
\hline Manuscript Type: & Article \\
\hline Date Submitted by the Author: & $16-$ Feb-2018 \\
\hline $\begin{array}{r}\text { Complete List of Authors: } \\
\text { Is the invited manuscript for } \\
\text { consideration in a Special } \\
\text { Issue? : }\end{array}$ & $\begin{array}{l}\text { OBrien, Eugene J.; University College Dublin, Civil Engineering } \\
\text { Offshore Structural Engineering } \\
\text { Malekjafarian, Abdollah; University College Dublin, Civil Engineering }\end{array}$ \\
\hline Keyword: & Wavelet, IRI, pavement, dynamic, deterioration \\
\hline \multicolumn{2}{|c}{} \\
\hline
\end{tabular}

SCHOLARONE

Manuscripts 


\section{An Alternative Roughness Index to IRI for Flexible Pavements}

3 Eugene J. OBrien ${ }^{1}$, Abdolrahim Taheri ${ }^{2}$, Abdollah Malekjafarian ${ }^{1 *}$

${ }^{1}$ School of Civil Engineering, University College Dublin, Dublin, Ireland.

6

$7 \quad{ }^{2}$ Department of Offshore Structural Engineering, Petroleum University of

8 Technology, Iran

9

10

11 *Corresponding author: Postal address: School of Civil Engineering, University

12 College Dublin, Newstead, Belfield, Dublin 4, Ireland.

13 Phone number: +35317163204.

14 Email address: abdollah.malekjafarian@ucd.ie

15

16 Word count: 5169

17

18

19

20

21

22 
39

40

\section{Abstract}

The International Roughness Index (IRI) is widely accepted as a measure of pavement condition. However it was developed as an indicator of passenger comfort as they travel in a vehicle on the pavement. In this paper, for the assumed failure model adopted, IRI is not found to be a good indicator of remaining pavement service life. The 3D continuous wavelet transform is proposed and shown to be a more effective indicator. Specific scales related to natural frequencies of the vehicle fleet, particularly the body mass frequency, are more significant than others. A weighted mean of the wavelet coefficients for these scales is used as an indicator of remaining life. 100 randomly generated class A profiles are generated and their histories of damage progression throughout their lives are simulated. The new indicator is applied to the initial profiles to determine which ones are more vulnerable to damage than others. In numerical simulations using the assumed damage model, the wavelet based indicator is shown to be well correlated with service life.

Keywords: Wavelet, IRI, pavement, dynamic, deterioration, damage, traffic.

\section{Introduction}

Road authorities need to routinely monitor flexible pavements to determine their condition in order to facilitate better maintenance planning. As a proxy of pavement condition, most developed countries measure the surface profiles of their main roads, typically with inertial profilers (Sayers and Karamihas 1996). Some studies have 
incorporated 3D laser-range sensors, video cameras and global positioning systems (GPS) to provide 3D reconstruction of road surfaces (Boyko and Funkhouser 2011; Huang et al. 2014; Yang et al. 2012; Yu et al. 2007). However, road profiles or surfaces have too much information to be used at a network level - at this level, a simple indicator of pavement condition is needed. Most road owners use the International Roughness Index (IRI), a single number that is intended to provide a measure of pavement condition. In this paper, it will be shown that IRI is not well correlated with pavement remaining service life and a better indicator is proposed.

The IRI is a widely used measure of the roughness of a longitudinal road surface profile. This index is obtained from the response of a specific quarter car model travelling on the pavement profile. It is a mathematical representation of the accumulated suspension stroke of the vehicle, divided by the distance travelled (Sayers 1989; Sayers and Karamihas 1996; Sun 2003). Du et al. (2014) propose a low-cost IRI measurement system using z-axis accelerometers and GPS. The relationship between IRI and Straightedge (SE)

61 Index is considered by Mucka (2012). SE can be calculated based on the measurement of 62 clearance between the road surface and a straightedge laid on it. Fuzzy and Gray theories are used in (Wang and Li 2011) for pavement roughness prediction. This study improves the regression they used to obtain the IRI of pavements. Sun (2003) proposed a method that can obtain the IRI of the pavement from the PSD of pavement roughness. The IRI can be simply calculated using the square root of the sum of the weighted regression coefficients of PSD roughness. 
69 Gonzalez et al. (2008) propose the use of vehicle acceleration for the estimation of road roughness. The resulting profile can be used for monitoring the pavement condition and predicting its remaining life. The profile can be transformed into the frequency domain using the Fourier transform and presented in the form of a Power Spectral Density (PSD) graph. The PSD can be used to describe the road roughness or as a basis for generating road profiles (Andrén 2005). PSD uses the Fourier transform to present the road profile as a function of its spatial frequencies (Lak et al. 2011; Ramji et al. 2004). Some researchers have investigated the relationship between PSD and the vehicle forces on pavements (Kropac and Mucka 2008; Labuschagne and Kululanga 2008; Lak et al. 2011). For example, Kropac and Mucka (2008) investigate the relation between PSD and road roughness on the one hand, and between dynamic vehicle response and the level of ground vibration on the other hand. Ambassa et al. (2013) investigate fatigue life prediction of an asphalt pavement subjected to multiple axle loadings using a viscoelastic finite element method.

PSD gives general information about frequency content but no spatial or time information (Brigham 1988: Pidwerbesky et al. 2006). Furthermore, it masks local features such as potholes. Wavelet analysis is a powerful method of transforming a signal in both the time and frequency domains simultaneously. In the analysis of longitudinal road profiles, time becomes distance and frequency is represented by spatial frequency. A wavelet transform computes the correlation between the signal and a selected wavelet function (Daubechies 1988: Lovász et al. 1986) for a range of frequencies for each point in space/time. Unlike the Fourier transform, wavelets 
preserve the spatial information of the original signal. The wavelet transform can be implemented in two ways: Continuous Wavelet Transform (CWT) and Discrete Wavelet Transform (DWT) (Newland 1993). In the context of road profiles, the Wavelet Transform can be used to determine the density of a range of frequencies at all points along the profile. Wei et al. use the Wavelet Transform as a tool to manage the pavement network (Wei et al. 2005). Papagiannakis et al. (2007) use DWT to interpret the dynamic axle load due to rubber and air suspension. They use the 5-axle semi-trailer with an air and a rubber suspension in the drive and trailer axles, respectively. They use the DWT to decompose the original signal into sub-bands to interpret the dynamic load.

In this paper, a CWT is used to determine the weighted mean of the intensities for a range of frequencies in a road profile which in turn give an indication of remaining pavement life. It is therefore proposed as the basis for a more appropriate roughness index than IRI when pavement damage is of interest. A truck fleet model is used to simulate millions of vehicles passing over the pavement. Initial IRI and wavelet transform are used for the prediction of remaining life of the pavement. It is shown that the road profile frequencies that are important for dynamic tyre forces are the natural frequencies that occur in the vehicle fleet. The hypothesis is that a profile with a high intensity of vehicle fleet frequencies is more vulnerable to damage and will deteriorate faster. It is also shown that the sum of the weighted mean squares of wavelet coefficient is well correlated with remaining pavement life and has the potential to provide more information compared to IRI. 


\section{Truck Fleet Model}

116 The pattern of dynamic load distribution plays an important role in the design and 117 assessment of flexible pavements. A large number of numerical truck model runs, is 118 usually employed for the assessment of flexible pavements (Belay et al. 2008). In this 119 paper, a quarter car model (Fig. 1) is used to simulate dynamic tyre forces which is 120 simple and widely used in the literature (Cebon 1999; Cole and Cebon 1992; Collop 121 and Cebon 1995; Turkay and Akcay 2005). Although, more complicated vehicle 122 models such as a half car can also be used, the quarter car is employed in this study

123 because it is the common vehicle normally used for calculating IRI in the literature. The 124 unsprung mass (representing the mass of the wheel and suspension) and sprung mass 125 (representing part of the mass of the vehicle body) are denoted $m_{u}$ and $m_{s}$ respectively.

126 The suspension system is represented by a linear spring of stiffness $k_{s}$ and a linear 127 damper with coefficient $c_{s}$, while the tyre is modelled by a linear spring of stiffness $k_{t}$ 128 and the road input irregularities are represented by $y_{r}$. Some of the more complex 129 behaviours that occur in real suspensions, e.g. the effect of suspension friction, have

130 been neglected here. It is acknowledged that the true behaviour of a suspension may be

131 different and that this could have a significantly impact on the response of the pavement 132 to a passing axle. 


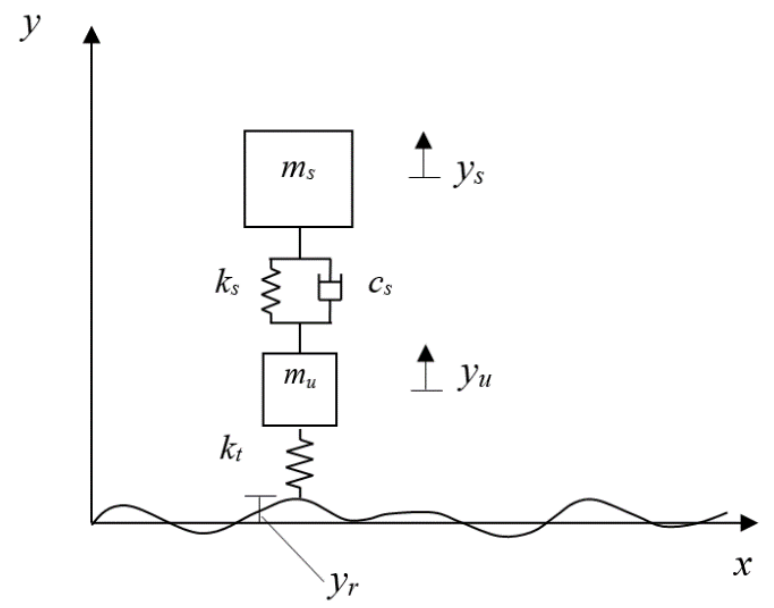

135

136 For the truck fleet models described here, all the vehicle parameter properties are 137 assumed to be Normally distributed, with the means and standard deviations taken from 138 the literature and given in Table 1 (Belay et al. 2008; Cebon 1999; Grave 2002; OBrien 139 and Enright 2011). The vehicle speed, v, is also assumed to be Normally distributed 140 with mean and standard deviation taken from measurement records for a highway site 141 on the RN10 near Angers, France (Grave 2002).

142 Table 1 - Vehicle parameters of quarter car fleet model

\begin{tabular}{|c|l|l|l|}
\hline No. & \multicolumn{1}{|c|}{ Vehicle parameter } & \multicolumn{1}{c|}{ Mean } & \multicolumn{1}{c|}{ Deviation } \\
\hline 1 & Unsprung mass, $m_{u}(\mathrm{~kg})$ & $420 \quad$ (Belay & 40 \\
& & et al. 2008; Cebon & (Belay et al. \\
& & $1999)$ & 2008) \\
\hline
\end{tabular}




\begin{tabular}{|c|c|c|c|}
\hline 2 & Sprung mass, $m_{s}(\mathrm{~kg})$ & $\begin{array}{l}4535 \\
\text { (OBrien and } \\
\text { Enright 2011) }\end{array}$ & $\begin{array}{l}500 \\
\text { (OBrien and } \\
\text { Enright 2011) }\end{array}$ \\
\hline 3 & Suspension stiffness, $k_{s}(\mathrm{~N} / \mathrm{m})$ & $\begin{array}{l}1000000 \text { (Belay } \\
\text { et al. 2008, Cebon } \\
\text { 1999) }\end{array}$ & $\begin{array}{l}100000 \\
\text { (Belay et al. } \\
2008)\end{array}$ \\
\hline 4 & Suspension damping, $c_{b}(\mathrm{Ns} / \mathrm{m})$ & $\begin{array}{l}20000 \quad \text { (Belay } \\
\text { et al. 2008; Cebon } \\
\text { 1999) }\end{array}$ & $\begin{array}{l}2,000 \\
\text { (Belay et al. } \\
2008)\end{array}$ \\
\hline 5 & Tyre stiffness, $k_{t}(\mathrm{~N} / \mathrm{m})$ & $\begin{array}{l}1950000 \text { (Belay } \\
\text { et al. 2008; Cebon } \\
\text { 1999) }\end{array}$ & $\begin{array}{l}200000 \\
\text { (Belay et al. } \\
2008)\end{array}$ \\
\hline 6 & Velocity, $v(\mathrm{~m} / \mathrm{s})$ & $\begin{array}{l}22.43 \quad \text { (Belay } \\
\text { et al. 2008; Grave } \\
\text { 2002) }\end{array}$ & $\begin{array}{l}2.40 \\
\text { (Grave 2002) }\end{array}$ \\
\hline
\end{tabular}

144

\section{Initial IRI and pavement permanent deformation}

146 The IRI is a profile-based roughness statistic that has become a standard indicator of

147 road roughness and ride comfort around the world (Sayers and Karamihas 1996).

148 Technically, IRI is a mathematical representation of the accumulated suspension stroke

149 of a specific vehicle travelling at $80 \mathrm{~km} / \mathrm{h}$, divided by the distance travelled along the

150 profile. The index is normally reported in $\mathrm{m} / \mathrm{km}$. In order to calculate the IRI, the quarter 
151 car parameters for masses, stiffnesses and damping are set to represent a reference car,

152 named the "Golden car". The parameters (normalized to $m_{s}=1$ ) for the Golden car are:

153

$$
c_{s}=6[1 / \mathrm{s}], \quad k_{t}=653\left[1 / \mathrm{s}^{2}\right], k_{s}=63.3\left[1 / \mathrm{s}^{2}\right], m_{u} / m_{s}=0.15
$$

155

156 To investigate the correlation between IRI and remaining pavement life, 200 simple

157 sine wave profiles are chosen with a range of frequencies. The amplitudes (A) are all

158 taken as $5 \mathrm{~mm}$, but the frequencies are changed from 0.01 cycles $/ \mathrm{m}$ to 2 cycles $/ \mathrm{m}$ in

159 steps of 0.01 cycles $/ \mathrm{m}$. The equation of motion for this two degree of freedom system 160 is:

161

$$
\left[\begin{array}{cc}
m_{s} & 0 \\
0 & m_{u}
\end{array}\right]\left[\begin{array}{l}
\ddot{y}_{s} \\
\ddot{y}_{u}
\end{array}\right]+\left[\begin{array}{cc}
c_{s} & -c_{s} \\
-c_{s} & c_{s}
\end{array}\right]\left[\begin{array}{l}
\dot{y}_{s} \\
\dot{y}_{u}
\end{array}\right]+\left[\begin{array}{cc}
k_{s} & -k_{s} \\
-k_{s} & k_{s}+k_{t}
\end{array}\right]\left[\begin{array}{l}
y_{s} \\
y_{u}
\end{array}\right]=\left[\begin{array}{c}
0 \\
y_{r} k_{t}
\end{array}\right]
$$

164 where $\ddot{y}_{s}$ and $\dot{y}_{s}$ are the nodal acceleration and velocity of the sprung mass, respectively

165 and $\ddot{y_{u}}$ and $\dot{y}_{\boldsymbol{u}}$ are the nodal acceleration and velocity of the unsprung mass, 166 respectively. The regular nature of the road profile results in a harmonic oscillation of 167 the quarter car. The dynamic component of the force exerted by the tyre $\left(\boldsymbol{F}_{\boldsymbol{y}}\right)$ is as 168 follows:

169

$$
\frac{F_{y}}{A}=k_{t} \omega^{2} \sqrt{\frac{\left(k_{t}\left(m_{s}+m_{u}\right)-m_{u} m_{u} \omega\right)^{2}+c_{s}^{2}\left(m_{s}+m_{u}\right) \omega^{2}}{f(\omega)^{2}+c_{s}^{2} \omega^{2} g(\omega)^{2}}}
$$


172 where

$173 \quad y_{r}=A \sin (\omega \mathrm{t})$

174

175

$$
f(\omega)=m_{s} m_{u} \omega^{4}-\left(k_{t} m_{s}+k_{s}\left(m_{s}+m_{u}\right)\right) \omega^{2}+k_{t} k_{s}
$$

176

$$
g(\boldsymbol{\omega})=\left(\boldsymbol{m}_{s}+\boldsymbol{m}_{u}\right) \boldsymbol{\omega}^{2}-\boldsymbol{k}_{t}
$$

180 The notional pavement has an asphalt thickness of $250 \mathrm{~mm}$. The stiffness modulus of

181 the asphalt layer is calculated assuming the following properties: annual range of mean

182 monthly air temperature $4^{0} \mathrm{C}$ to $18^{0} \mathrm{C}$, void content $10 \%$, binder content $3.5 \%$, Specific

183 Gravity of binder $2700 \mathrm{~kg} / \mathrm{m}^{3}$, Specific Gravity of aggregate $1020 \mathrm{~kg} / \mathrm{m}^{3}$ and proportion

184 of binder $7.9 \%$. The granular layer has a modulus of elasticity of $400 \mathrm{MPa}$ and is 200

185 mm thick. The subgrade layer is assumed to be infinitely thick with a modulus of 50

$186 \mathrm{MPa}$.

187 A mechanistic-empirical approach, proposed by Collop and Cebon (1995), is used to

188 assess the remaining service life of the pavement. The model has been used extensively

189 in the literature (Bilodeau et al. 2017; Goodrum and Cebon 2016; Malekjafarian et al.

190 2017) and validated using real measurements by De-Pont et al. (2004). This method

191 calculates pavement damage in the form of permanent deformation and loss of stiffness

192 through fatigue, although there may be other sources of pavement deterioration e.g.

193 freeze/thaw. The pavement is deemed to have reached the end of its service life when

194 it reaches either of these two limit states. The permanent deformation (rutting) 
195 influences the vehicle/pavement dynamic interaction and a feedback loop is necessary 196 to periodically update the profile and recalculate the pattern of applied forces. The 197 process of accumulating damage in the road profile is repeated for millions of wheel 198 passes until the pavement has reached the end of its serviceable life, defined here as 199 when the accumulated permanent deformation reaches $20 \mathrm{~mm}$. Details of the model are 200 outlined in (OBrien and Taheri 2012).

201 Monte Carlo simulation is used to find the relative number of occurrences of each 202 natural frequency in the quarter car fleet. The simulated speeds are used to convert the 203 time frequencies to spatial frequencies. Fig. 2 shows the distribution for each spatial 204 natural frequency in the fleet.

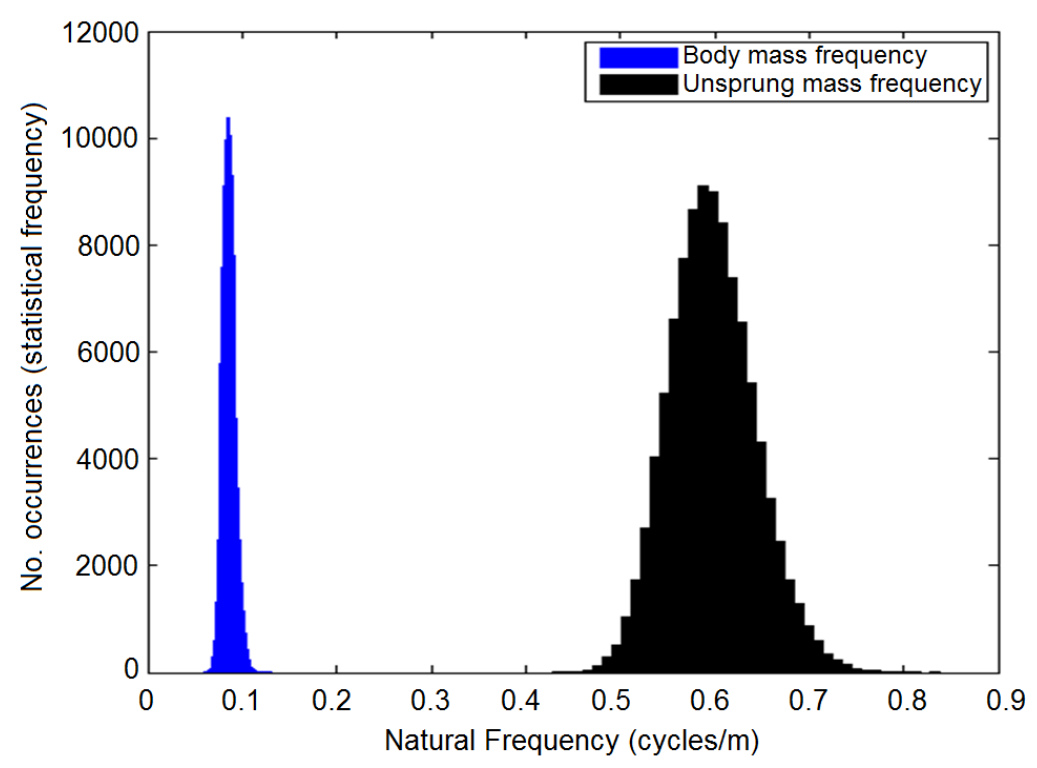

Figure 2 - Quarter car fleet spatial frequencies

206 Vehicles from this fleet are run 10 million times over each of the 200 sine wave profiles.

207 The permanent deformation of the pavement for each sine wave is calculated (Fig. 3). 
208 It can be seen in this figure that the pavements with natural frequencies near those of the fleet $(0.08$ and 0.595 cycles $/ \mathrm{m})$, are subject to resonance and hence greater damage.

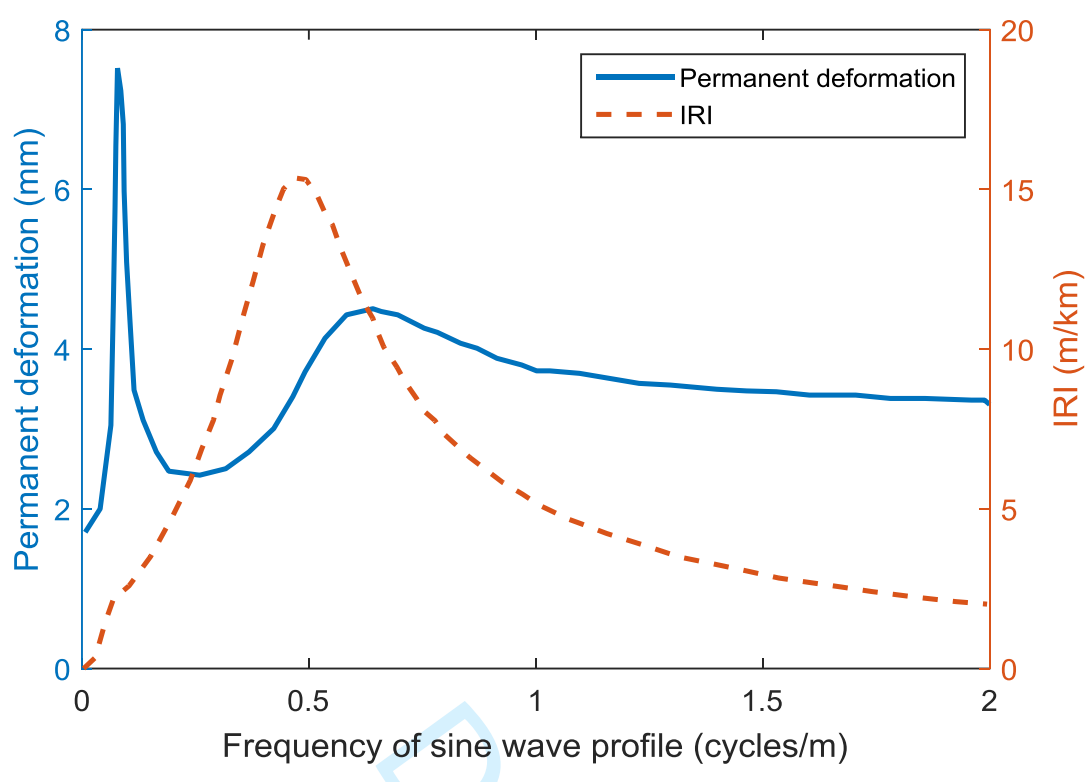

211 Figure 3 - Permanent deformation and IRI for 200 different sine wave profiles

213 IRI is also calculated when the Golden Car vehicle, which has frequencies of 0.054 and

2140.495 cycles/m, runs over 200 sine waves (Fig. 3). The Golden Car is excited by the 215 sine wave profiles which have frequencies closest to its frequencies. It is shown in Fig. 2163 that there is a small peak on IRI at the first frequency but a dominant peak at the 217 second unsprung mass frequency. It can be seen that those road profiles that have high 218 IRI are, in general, not those with greater permanent deformation.

219 The permanent deformation for each sine wave profile is plotted versus IRI in Fig. 4. 220 There is a peak in damage at a road profile frequency of $f=0.08$ cycles $/ \mathrm{m}$, when IRI is 221 quite low (about $2.1 \mathrm{~m} / \mathrm{km}$ ). The damage then falls and remains reasonably constant, as 222 IRI increases, until a road profile frequency of about $f=0.31$ cycles $/ \mathrm{m}$. There is a region 
223 in the graph after this when both damage and IRI increase until IRI reaches its peak at $224 f=0.495$ cycles $/ m$. After this, damage continues to increase, as IRI falls until it peaks 225 at $f=0.595$ cycles $/ \mathrm{m}$. Beyond that point, both damage and IRI fall.

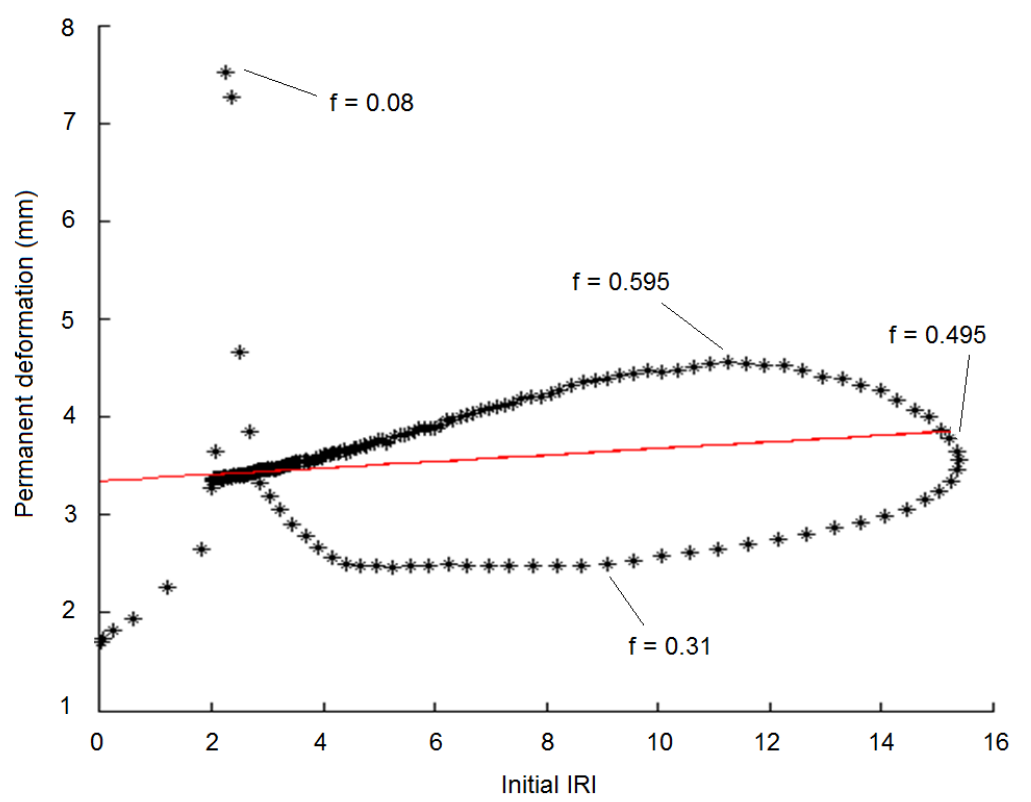

Figure 4 - Correlation between initial IRI and permanent deformation for 200

230 It might be expected that an initially smooth pavement (low IRI) would have little

231 damage while a rough pavement would have greater damage. However, it is clear from

232 Fig. 3 that different pavement frequencies influence damage and IRI. Fig. 4 confirms

233 that the correlation between damage and IRI is poor.

235 4. Wavelet Transform as a damage index

$236 \quad 4.1$ Wavelet Transform of contact force 
237 It is assumed here that the natural frequencies of the fleet are excited by the road profile

238 and that the presence of these frequencies in the profile makes it more susceptible to

239 high dynamic forces and hence to damage. Therefore, it is important to know at which

240 points along the profile, the natural frequencies of the fleet are excited most. The

241 Wavelet Transform represents a signal in the time and frequency domains. It uses a

242 mother wavelet which can have many different forms. The Daubechies $3(\mathrm{db} 3)$ mother

243 wavelet is chosen in this study as it is found to provide adequate resolution in both

244 frequency and time (distance) simultaneously (Papagiannakis et al. 2007; Wei et al.

245 2005). A randomly generated class A initial road profile is generated with a sample

246 interval of $100 \mathrm{~mm}$ (Fig. 5). A vehicle with the mean fleet properties (Table 1) is

247 simulated to pass over this profile. The contact force between the vehicle and the

248 pavement is calculated using Eq. 3 (Fig. 6).

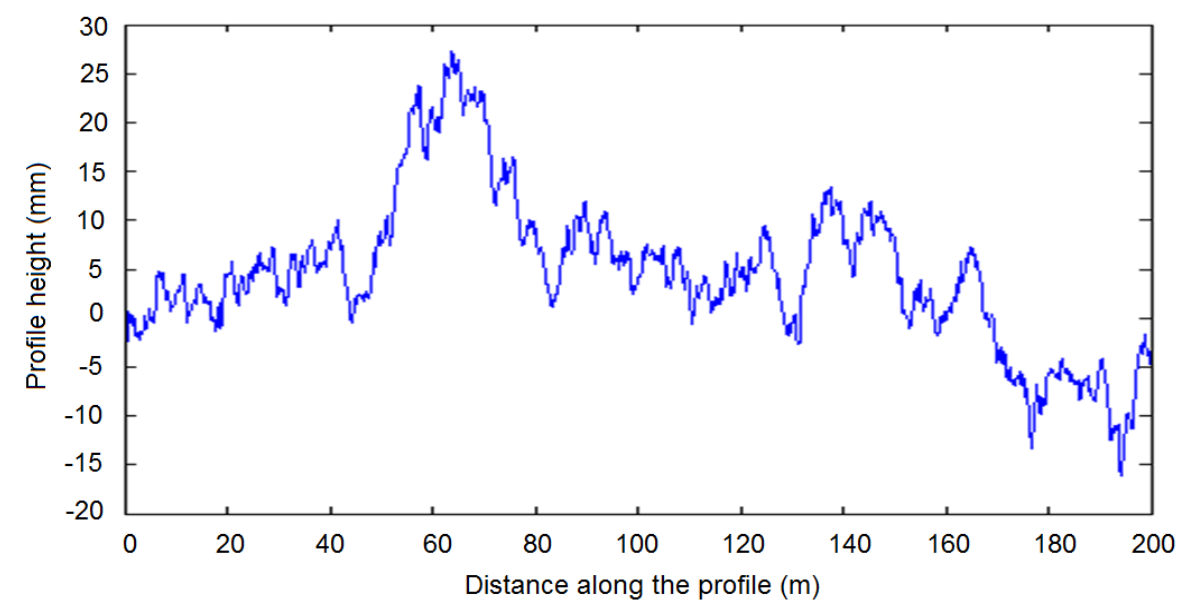

Figure 5 - Randomly generated Class A initial profile 


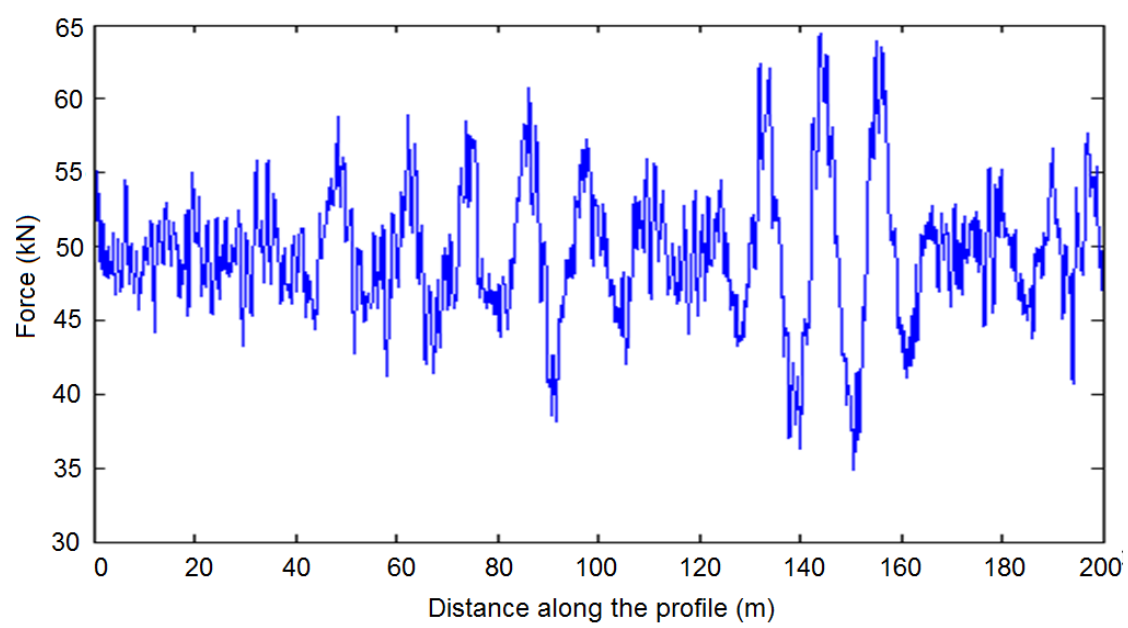

Figure 6 - Contact force on initial profile.

253 The CWT of the contact force pattern is shown in Fig. 7(a). The scale axis in the CWT 254 corresponds to the inverse of frequency while the Wavelet coefficient is a kind of 255 density for any given scale and point in time or space. The fleet frequencies are, from 256 Fig. 2, around $f=0.595$ and $f=0.085$ cycles $/ \mathrm{m}$. The corresponding scale values are 14 257 and 94 respectively. Cross sections through the CWT at these scales are shown in Figs. 258 7(b) and 7(c). The CWT of the force signal shows high density around the important 259 body mass frequency (scale $=94$ ) at distances along the profile such as $x=145 \mathrm{~m}$. It is 260 therefore expected that damage will accumulate in these parts of the profile until the 261 pattern of forces changes. 


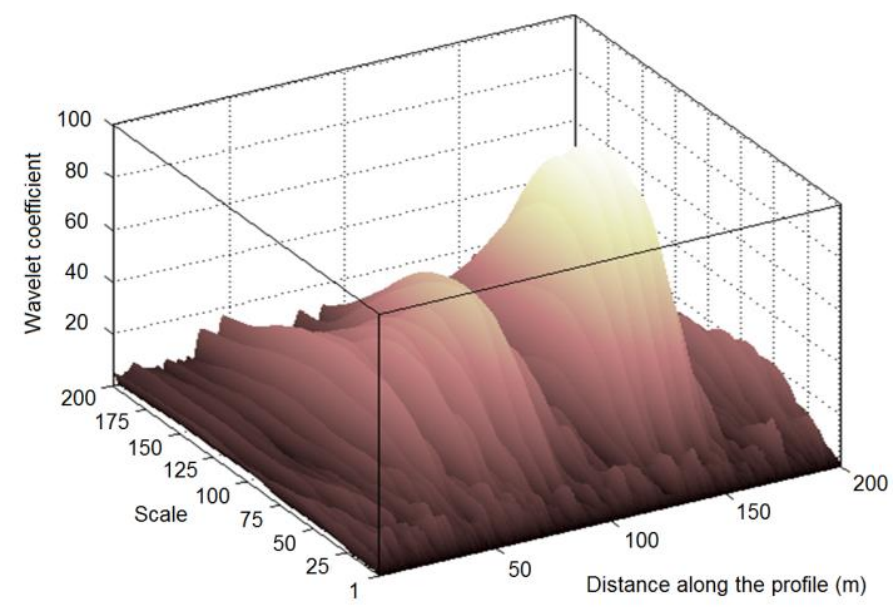

(a)

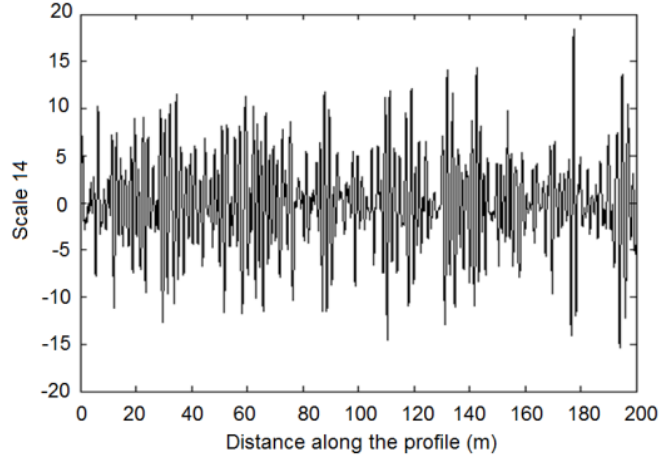

(b)

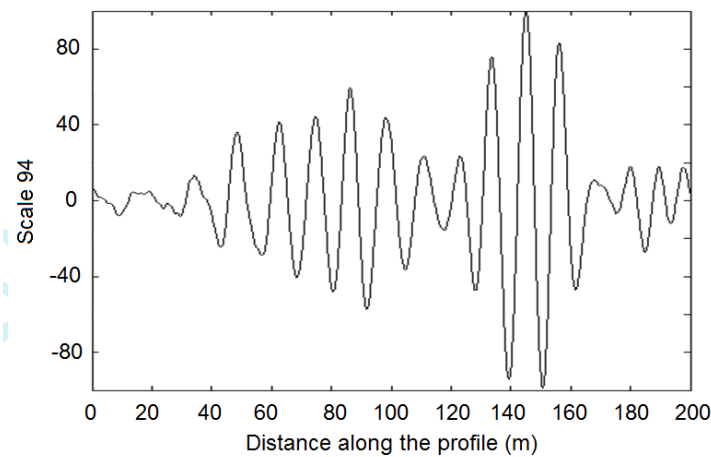

(c)

262 Figure 7 - Time history and CWT of contact force, (a) Wavelet transform of force

263 (absolute values of coefficients), (b) Scale $14(f=0.595)$ and (c) Scale $94(f=0.085)$.

\subsection{Wavelet transform of the profile}

266 The changes in the road profile after 10 and 15 million axles have passed, are calculated

267 using the method explained in (Taheri et al. 2012). The CWT is applied to the initial

268 and two changed profiles. Fig. 8 shows the wavelet coefficients for the profiles. 
(a)

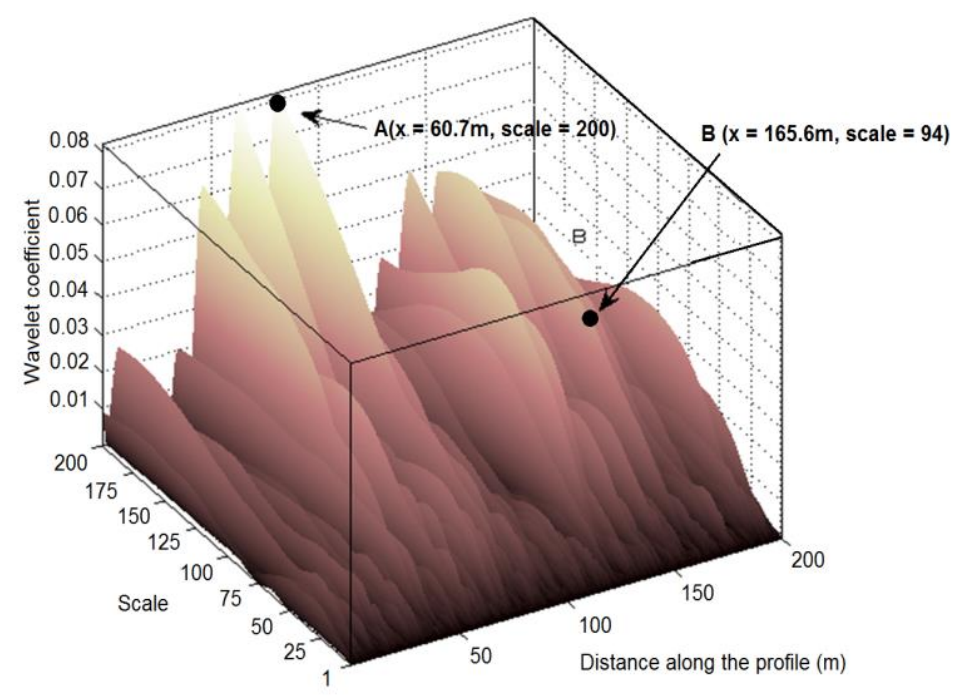

(b)

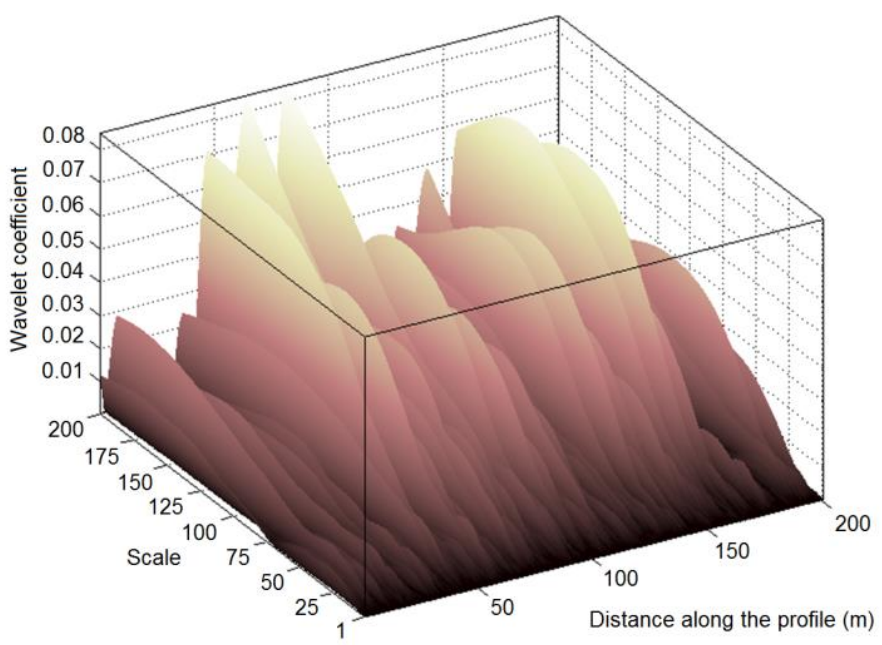


(c)

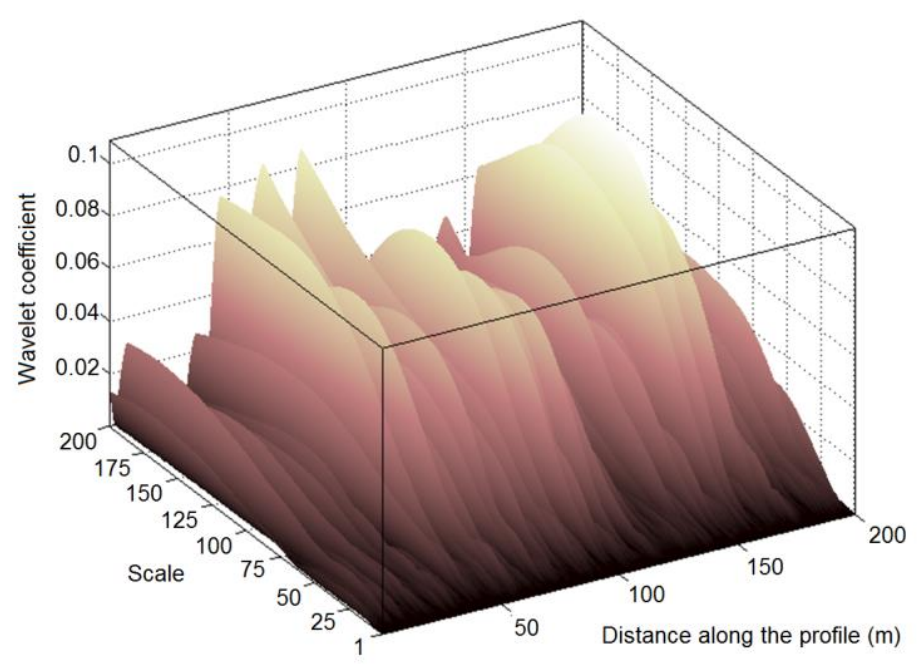

270 Figure 8-CWT of changing road profile (absolute values of coefficients), (a) Initial profile, (b) Profile after 10 million axles and (c) Profile after 15 million axles.

273 The important scales are those corresponding to the fleet body mass and unsprung mass

274 frequencies. Using the relative number of occurrences in Fig. 2, a weighted mean of the

275 Wavelet coefficients around the body mass frequency is calculated and plotted in Fig.

276 9. The Peak A in Fig. 8(a) (at about $x=80.6 \mathrm{~m}$ ) does not occur at a critical scale. It 277 therefore does not appear in Fig. 9 and does not grow greatly as pavement damage 278 progresses (see Figures 8 (b) and (c)). On the other hand Peak B in Fig. 8(a) does occur 279 near a critical frequency and a corresponding peak is apparent at $x=165.2 \mathrm{~m}$ in Fig. 9.

280 The peak in the CWT plot suggests a likelihood of resonance and correctly predicts 281 damage near this point. The CWT is not always perfect however. 


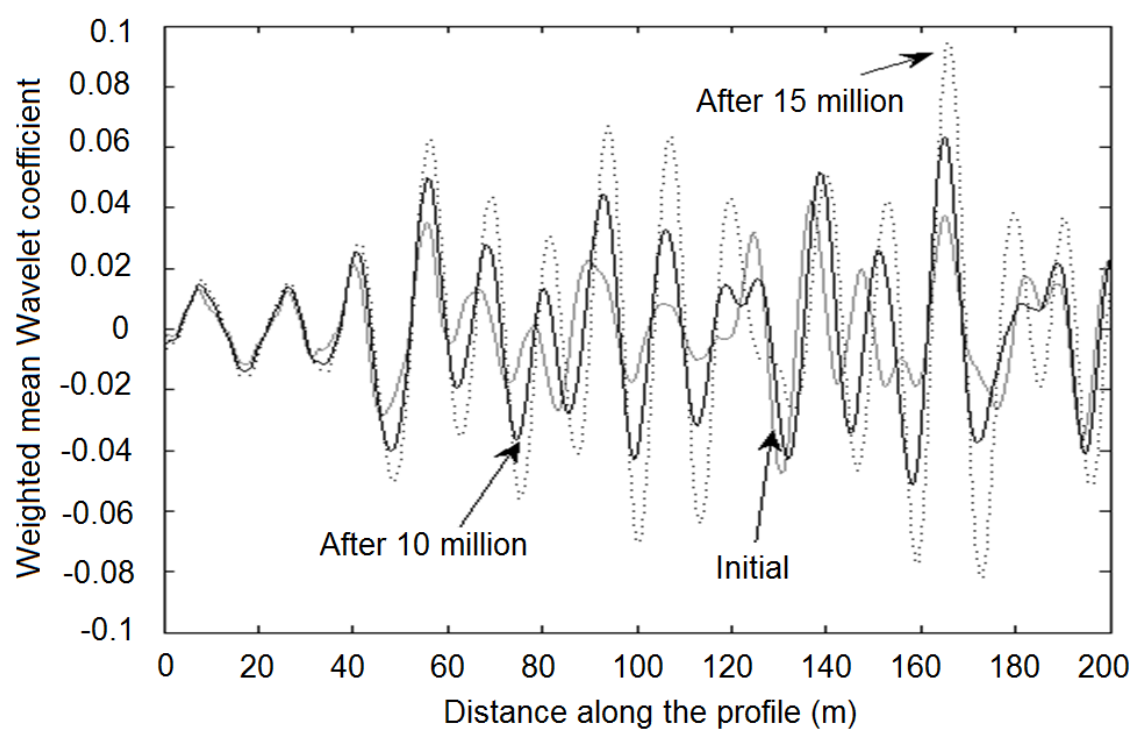

284 Figure 9 - Weighted mean of Wavelet coefficients corresponding to body mass frequency

287 The corresponding permanent deformations are shown in Fig. 10. At about $x=140 \mathrm{~m}$, 288 Fig. 10 shows a relatively high density, but the magnitude of the peak force does not 289 develop at this point with increasing number of axles. However, the pavement 290 deformation (Fig. 10) is large near both $x=140 \mathrm{~m}$ and $x=165.2 \mathrm{~m}$. 


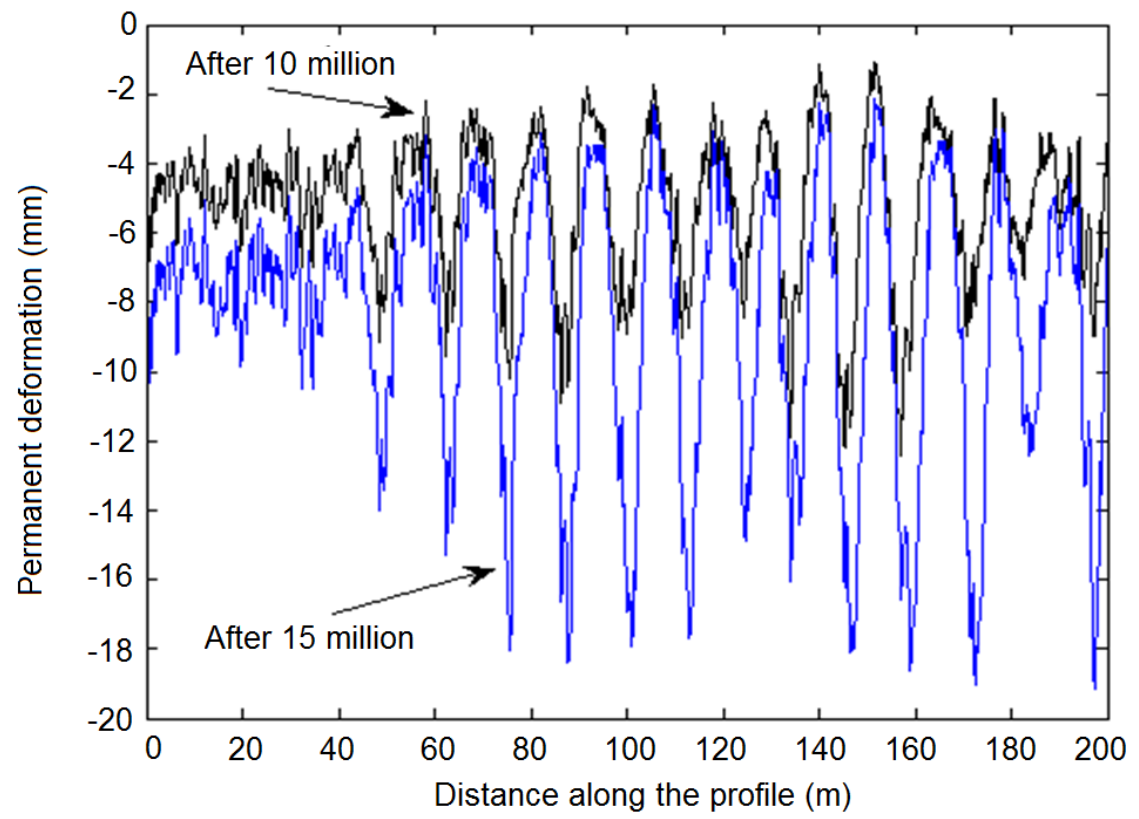

Figure 10 - Permanent deformation (after 10 and 15 million quarter cars)

\section{Prediction of remaining pavement life}

\subsection{Sine wave profiles}

296 As it is the absolute value of wavelet coefficient that indicates strong correlation with

297 the mother wavelet, an energy term that is always positive is defined:

wavelet energy $=\sum_{i=1}^{n_{f}} \sum_{j=1}^{n_{p}} a_{i} s_{i j}^{2}$

302 where $s_{\mathrm{ij}}$ is the wavelet coefficient at scale/frequency $i$ and point $j$ along the profile, $a_{\mathrm{i}}$

303 is the relative number of occurrences of scale/frequency $i$ in the fleet and $n_{\mathrm{f}}$ and $n_{\mathrm{p}}$ are 304 the numbers of scales/frequencies and points respectively. In this case, the 
305 scales/frequencies include only the body mass frequencies as Fig. 3 indicates that these 306 are the more critical for permanent deformation. The same 200 simple sine wave 307 profiles as were used to test IRI are used in this section to test wavelet energy as a 308 predictor of remaining service life. It can be seen in Figures 2 and 3 that damage occurs 309 in profiles other than those with the fleet frequencies but that damage is maximum for 310 the body mass frequency. The results are shown in Fig. 11 where it can be seen that 311 there is a good correlation between maximum permanent deformation and wavelet 312 energy. There are a number of outlying points but these pure sine waves are atypical 313 profiles. Further, the correlation is much better than it was with initial IRI (Fig. 4).

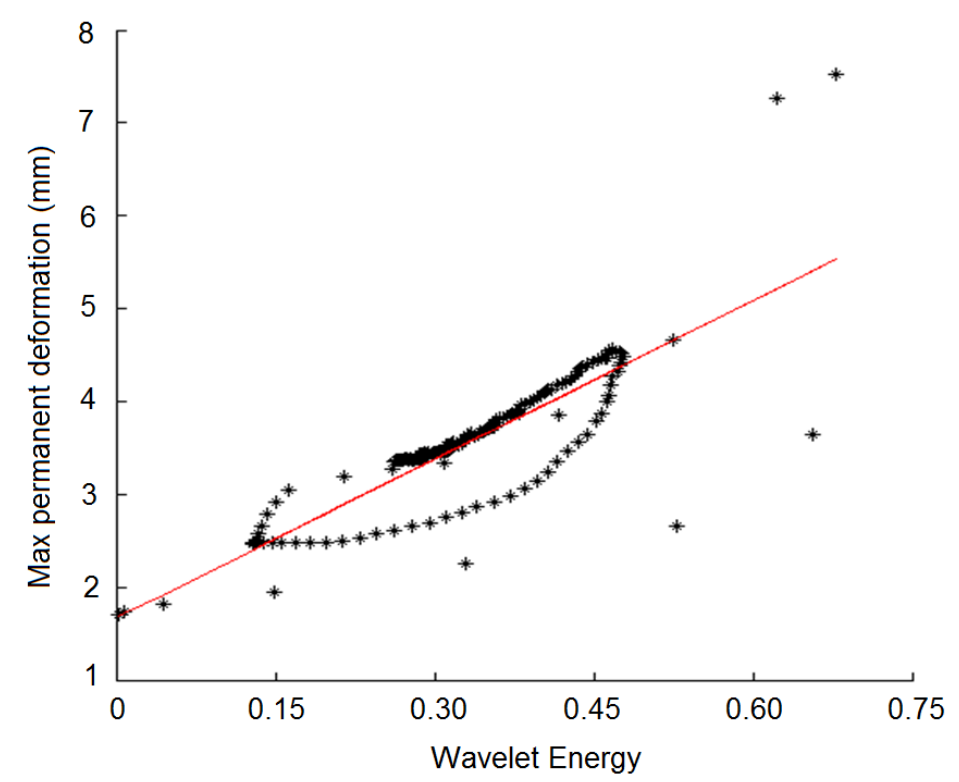

Figure 11 - Correlation between wavelet parameter and maximum permanent deformation for 200 different sine wave profiles after 10 million axles (red line: linear regression). 


\subsection{Random profiles}

317 To develop a predictor of remaining pavement life, 100 randomly generated class A 318 (Malekjafarian and OBrien 2014) initial road profiles are generated that are $200 \mathrm{~m}$ long 319 with a sample interval of $100 \mathrm{~mm}$. Due to the fact that discontinuities occur at the edges 320 of a road signal, a "Hanning window" is used at the edge. (The Hanning window 321 attenuates the elevations near the edges, while leaving intact the profile values near the 322 middle).

323 The mechanistic-empirical approach is again used to assess the remaining service life 324 of the pavement for each profile. Fig. 12 shows initial IRI and maximum permanent 325 deformation after 25 million passes of quarter cars from the fleet (Table 1), for each of 326 the 100 profiles. The permanent deformation ranges from about $5.5 \mathrm{~mm}$ up to about 19 $327 \mathrm{~mm}$. As can be seen, there is some correlation with initial IRI, showing that it is a 328 reasonable predictor of remaining life. The correlation coefficient is 0.66 .

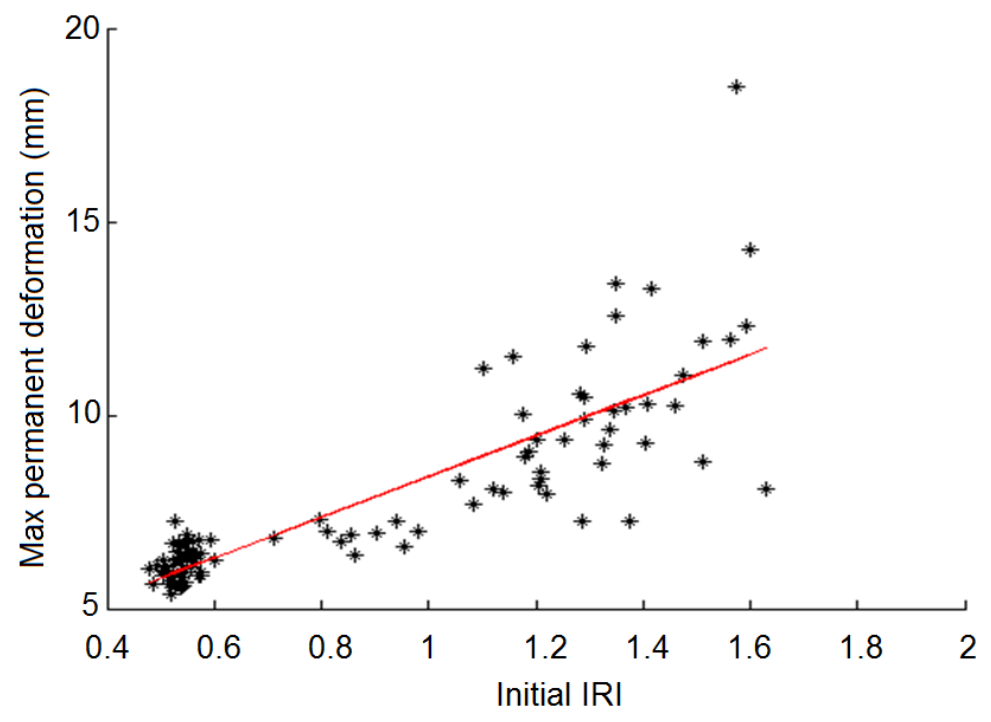


Figure 12 - Correlation between initial IRI and maximum permanent deformation after 25 million axles (red line: linear regression).

330 The same 100 randomly generated class A initial road profiles are also used to test

331 wavelet energy as a predictor of remaining service life. The wavelet energy is calculated 332 for each scale by summing, at $100 \mathrm{~mm}$ intervals, throughout the $200 \mathrm{~m}$ length of each 333 profile and taking the weighted mean around the vehicle fleet scales/frequencies. Fig. 33413 shows the initial wavelet energy and the maximum permanent deformation after 25 335 million passes of quarter cars for each of the 100 profiles. As can be seen, there is good 336 correlation, showing that wavelet energy is a good predictor of remaining life. The 337 correlation coefficient is 0.92 , as opposed to 0.66 for the IRI. There are several possible 338 reasons for this improvement. The IRI is calculated using a reference quarter car, while 339 a truck fleet is employed for the alternative approach. The output suspension stroke rate 340 of the reference quarter car is considered for IRI which gives the average rectified value, 341 but the proposed method uses tire load variation to estimate the wavelet energy. Further 342 investigations, e.g. optimization of the golden car properties, may improve the 343 correlation factor for that approach. 


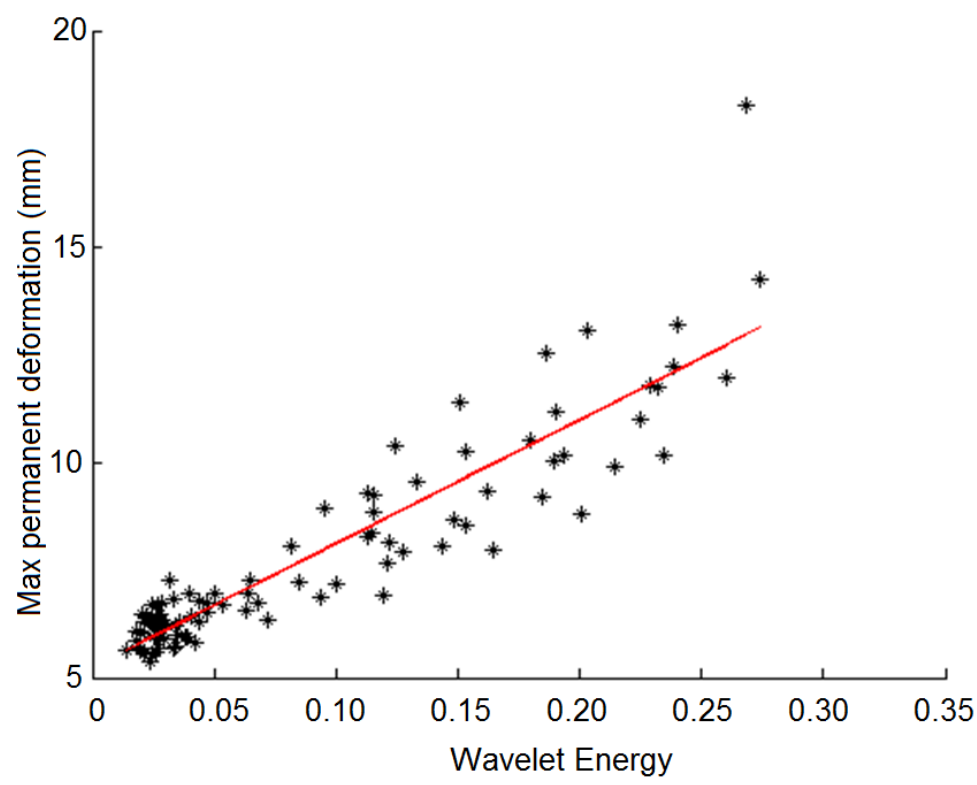

Figure 13 - Correlation between wavelet parameter and maximum permanent deformation after 25 million axles (red line: linear regression).

\section{Conclusions and discussion}

346 This paper investigates the effectiveness of a Continuous Wavelet Transform (CWT)

347 parameter as an indicator of remaining pavement life. Truck fleet frequencies are

348 shown to be important for pavement damage but significant damage also occurs in

349 simple sine wave profiles with other frequencies. Nevertheless, a wavelet energy

350 parameter that sums the weighted mean squares of wavelet coefficients is shown to be

351 well correlated with remaining pavement life. The weighting is based on the relative

352 numbers of occurrences of natural frequencies in the vehicle fleet.

353 For more general application, the accuracy of the assumed distribution of body mass

354 frequencies in the vehicle fleet will reduce the effectiveness of the predictor although

355 there is some evidence to suggest that these can be extracted from multiple-sensor 
356 Weigh-in-Motion systems. Further, the correlation can be expected to be weaker when non-vehicle sources of damage such as extreme weather events are considered. The new wavelet energy parameter is a structural performance indicator which performs better that the current IRI and would appear to have good potential as an indicator of remaining pavement life. However, performance of the new indicator needs to be 361 validated using field measurements.

\section{Acknowledgements}

364 The authors acknowledge the support received from the European Framework project,

Science Foundation Ireland supporting this research under the US/Ireland programme.

\section{References}

Ambassa, Z., Allou, F., Petit, C., and Eko, R.M. 2013. Fatigue life prediction of an asphalt pavement subjected to multiple axle loadings with viscoelastic FEM. Constr Build Mater 43: 443-452.

Andrén, P. 2005. Power spectral density approximations of longitudinal road profiles. International Journal of Vehicle Design 40: 2-14.

Belay, A., OBrien, E., and Kroese, D. 2008. Truck fleet model for design and assessment of flexible pavements. Journal of Sound and Vibration 311(3-5): 1161-1174.

Bilodeau, J.P., Gagnon, L., and Dore, G. 2017. Assessment of the relationship between the international roughness index and dynamic loading of heavy vehicles. Int J Pavement Eng 18(8): 693-701.

Boyko, A., and Funkhouser, T. 2011. Extracting roads from dense point clouds in large scale urban environment. Isprs J Photogramm 66(6): S2-S12.

Brigham, E.O. 1988. The Fast Fourier Transform and its applications. Prentice Hall: Englewood Cliffs, New Jersey, USA.

Cebon, D. 1999. Handbook of vehicle-road interaction.

Cole, D., and Cebon, D. 1992. Spatial repeatability of dynamic tyre forces generated by heavy vehicles. Proceedings of the Institution of Mechanical Engineers, Part D: Journal of Automobile Engineering 206(1): 17-27. 
Collop, A., and Cebon, D. 1995. A model of whole-life flexible pavement performance. Proceedings of the Institution of Mechanical Engineers, Part C: Journal of Mechanical Engineering Science 209(6): 389407.

Daubechies, I. 1988. Orthonormal bases of compactly supported wavelets. Communications on pure and applied mathematics 41(7): 909-996.

De-Pont, J., Thakur, K., and Pidwerbesky, B. 2004. Validating a whole life pavement performance model. Transit New Zealand, CAPTIF Research Facility.

Du, Y.C., Liu, C.L., Wu, D.F., and Jiang, S.C. 2014. Measurement of International Roughness Index by Using Z-Axis Accelerometers and GPS. Math Probl Eng.

Gonzalez, A., O'Brien, E.J., Li, Y.Y., and Cashell, K. 2008. The use of vehicle acceleration measurements to estimate road roughness. Vehicle Syst Dyn 46(6): 485-501.

Goodrum, W.J., and Cebon, D. 2016. Synthesising spatially repeatable tyre forces from axle load probability distributions. P I Mech Eng C-J Mec 230(5): 699-714.

Grave, S. 2002. Modelling of site-specific traffic loading on short to medium span bridges, Trinity College Dublin.

Huang, J.P., Liu, W.Y., and Sun, X.M. 2014. A Pavement Crack Detection Method Combining 2D with 3D Information Based on Dempster-Shafer Theory. Comput-Aided Civ Inf 29(4): 299-313.

Kropac, O., and Mucka, P. 2008. Effect of obstacles in the road profile on the dynamic response of a vehicle. P I Mech Eng D-J Aut 222(D3): 353-370.

Labuschagne, F., and Kululanga, G.K. 2008. An overview of the neural network based technique for monitoring of road condition via reconstructe4d road profiles. SATC 2008.

Lak, M.A., Degrande, G., and Lombaert, G. 2011. The effect of road unevenness on the dynamic vehicle response and ground-borne vibrations due to road traffic. Soil Dyn Earthq Eng 31(10): 1357-1377.

Lovász, L., Cushing, J., Kurtz, T.G., Fornberg, B., Flyer, N., Winograd, S., Salton, G., Fokas, A.S., Lindley, D., and Sattinger, D. 1986. CBMS-NSF Regional Conference Series in Applied Mathematics. SIAM.

Malekjafarian, A., and OBrien, E.J. 2014. Identification of bridge mode shapes using Short Time Frequency Domain Decomposition of the responses measured in a passing vehicle. Engineering Structures 81: 386-397.

Malekjafarian, A., Martinez, D., and O'Brien, E.J. 2017. Pavement Condition Measurement at High Velocity using a TSD, CRC Press.

Mucka, P. 2012. Relationship between International Roughness Index and Straightedge Index. J Transp Eng-Asce 138(9): 1099-1112.

Newland, D. 1993. Random Vibrations, Spectral \& Wavelet Analysis, Wesley Longman. Inc.

OBrien, E.J., and Enright, B. 2011. Modeling same-direction two-lane traffic for bridge loading. Struct Saf 33(4-5): 296-304.

OBrien, E.J., and Taheri, A. 2012. Numerical integration approach to the problem of simulating damage in an asphalt pavement. International Journal of Pavement Engineering 13(4): 339-349.

Papagiannakis, A.T., Zelelew, H.M., and Muhunthan, B. 2007. A wavelet interpretation of vehiclepavement interaction. International Journal of Pavement Engineering 8(3): 245-252.

Pidwerbesky, B., Waters, J., Gransberg, D., and Stemprok, R. 2006. Road surface texture measurement using digital image processing and information theory. Land Transport New Zealand Research Report 290: 65-79.

Ramji, K., Gupta, A., Saran, V., Goel, V., and Kumar, V. 2004. Road roughness measurements using PSD approach. Journal of the Institution of Engineers. India. Civil Engineering Division 85(nov): 193201.

Sayers, M.W. 1989. Two quarter-car models for defining road roughness: IRI and HRI. Transportation Research Record(1215).

Sayers, M.W., and Karamihas, S.M. 1996. Interpretation of road roughness profile data Final Report, Federal Highway administration. The University of Michigan Transportation Research Institute (UMTRI).

Sun, L. 2003. Simulation of pavement roughness and IRI based on power spectral density. Math Comput Simulat 61(2): 77-88. 
440 Taheri, A., OBrien, E.J., and Collop, A.C. 2012. Pavement damage model incorporating vehicle 441 dynamics and a 3D pavement surface. International Journal of Pavement Engineering 13(4): 374-383.

442 Turkay, S., and Akcay, H. 2005. A study of random vibration characteristics of the quarter-car model. 443 Journal of Sound and Vibration 282(1-2): 111-124.

444 Wang, K.C.P., and Li, Q.A. 2011. Pavement Smoothness Prediction Based on Fuzzy and Gray Theories. 445 Comput-Aided Civ Inf 26(1): 69-76.

446 Wei, L., Fwa, T.F., and Zhe, Z. 2005. Wavelet analysis and interpretation of road roughness. J Transp 447 Eng-Asce 131(2): 120-130.

448 Yang, B.S., Fang, L.N., Li, Q.Q., and Li, J. 2012. Automated Extraction of Road Markings from Mobile 449 Lidar Point Clouds. Photogramm Eng Rem S 78(4): 331-338.

450 Yu, S.J., Sukumar, S.R., Koschan, A.F., Page, D.L., and Abidi, M.A. 2007. 3D reconstruction of road 451

452

453 
470 List of Tables:

471 Table 1 - Vehicle parameters of quarter car fleet model

472

473

474

475

476

477

478

479

480

481

482

483

484

485

486

487

488

489

490

491

492 
List of Figures:

494 Figure 1 - Linear two-degree of freedom quarter car model

495 Figure 2 - Quarter car fleet spatial frequencies

496 Figure 3 - Permanent deformation and IRI for 200 different sine wave profiles

497 Figure 4 - Correlation between initial IRI and permanent deformation for 200

498 different sine wave profiles after 10 million quarter cars (red line: linear regression).

499 Figure 5 - Randomly generated Class A initial profile

$500 \quad$ Figure 6 - Contact force on initial profile.

501 Figure 7 - Time history and CWT of contact force, (a) Wavelet transform of force

502 (absolute values of coefficients), (b) Scale $14(f=0.595)$ and (c) Scale $94(f=0.085)$.

503 Figure 8-CWT of changing road profile (absolute values of coefficients), (a) Initial

504 profile, (b) Profile after 10 million axles and (c) Profile after 15 million axles.

505 Figure 9 - Weighted mean of Wavelet coefficients corresponding to body mass

506 frequency

507 Figure 10 - Permanent deformation (after 10 and 15 million quarter cars)

508 Figure 11 - Correlation between wavelet parameter and maximum permanent

509 deformation for 200 different sine wave profiles after 10 million axles (red line: linear

510 regression).

511 Figure 12 - Correlation between initial IRI and maximum permanent deformation

512 after 25 million axles (red line: linear regression).

513 Figure 13 - Correlation between wavelet parameter and maximum permanent

514 deformation after 25 million axles (red line: linear regression). 